Revista Hechos y Proyecciones del Lenguaje. No. 12. Universidad de Nariño -UNED. (ISSN: 0121-3350). (pp. 25-34). 2003.

APA Citation Style: Benavides B., Jorge E. (2003). Cambios Léxico-semánticos del Inglés al Español en el Argot de la Informática. Revista Hechos y Proyecciones del Lenguaje (12), 25-34.

\title{
Cambios Lexico-semánticos del Inglés al Español en el Argot de la Informática
}

\author{
Jorge E. Benavides B. (joelbebu@gmail.com) \\ Departamento de Lingüística e Idiomas \\ Universidad de Nariño, San Juan de Pasto, Colombia
}

\section{INTRODUCCION}

En el siguiente artículo se intentará determinar algunos de los cambios lingüísticos producidos en nuestro medio debido a la introducción en forma decisiva del campo de la computación e informática en las diferentes labores de la vida diaria y principalmente en el campo académico. Estos procesos lingüísticos de tipo lexical y semántico hacen parte de los procesos de cambio de orden sincrónico y diacrónico que se pueden atestiguar en la actualidad y que cumplen un recorrido histórico de cambio lingüístico.

\section{La revolución de la informática}

La informática y sus aplicaciones en nuestros días, de bastante difusión en nuestro medio están causando cambios en el repertorio lingüístico de la gente, tanto en el campo escrito como oral. Esto se puede notar entre los integrantes de un grupo de personas con características afines de trabajo y especialidad que comparten un "dialecto", argot o jerga en el que hacen uso de términos que se han introducido del Inglés al Español en variedad de formas: forma directa u original, traducciones literales al Español, alteraciones de forma y la formación de nuevos términos a partir de los mismos términos extranjeros.

A continuación se exponen algunos de los muchos términos que a diario se escuchan y se leen (en la literatura de la informática) por el cada día más creciente grupo de usuarios de la computación y la informática.

1. Vamos a CORRER el programa

2. espere a que aparezca el PROMPT

3. utilice el COMANDO PRINT

4. Cuántas FILAS se copiaron?

5. ENTRE el siguiente comando 
Revista Hechos y Proyecciones del Lenguaje. No. 12. Universidad de Nariño -UNED. (ISSN: 0121-3350). (pp. 25-34). 2003.

6. antes que nada, SALVE su archivo

7. posicione el CURSOR en la palabra

8. Primero asegúrese de que el SOFTWARE sea para este HARDWARE

9. Hay que CARGAR el programa

10. EJECUTE el programa

11. La RUTINA INICIALIZA las variables

12. La SENTENCIA es incorrecta

13. ¿Alguien tiene un DOS?

14. Podemos TIPIAR el texto primero

15. Se te olvidó RETURNEAR

16. La orden debe ESPULEAR los archivos

17. Hay que FORMATEAR o INICIALIZAR este disco

Algunos de los términos señalados en la lista anterior causarían algún grado de duda sobre lo que significan o pueden significar en una primera lectura por un lector desprevenido. Sin embargo, esto no sucede con quienes están sumergidos en el argot de la tecnología informática. La tecnología hace que cada nueva introducción sea causa de la proliferación de términos y palabras técnicas que van poco a poco adentrándose en el repertorio formal e informal de los hablantes de las lenguas donde que se realiza una introducción tecnológica. Hoy en día hay cientos de palabras introducidas no solamente al español sino a los demás idiomas involucrados en el desarrollo de la tecnología de la información, la informática y la computación.

No obstante, esa 'normal' o 'natural' introducción de términos nuevos de un idioma a otro pueden causar interferencia semántica con el uso (Allan, 1986), lo cual incide en algunos de los aspectos lexico-semánticos de la lengua objeto. Por ejemplo, hay un aspecto interesante que tiene lugar entre los hablantes de una lengua quienes tienen que utilizar a menudo esta nueva terminología a pesar de que algunos de esos términos no tienen una equivalencia exacta al idioma en cuestión. No existe ninguna clase de impedimento para que ellos hagan uso de lo que se relacionaría a nivel lexicosemántico con la producción de oraciones nuevas en lo que Chomsky $(1974,74)$ llama 'creatividad lingüística' o creatividad del lenguaje: “... la habilidad del hablante de producir nuevas oraciones, oraciones que son comprendidas inmediatamente por otros hablantes a pesar de no poseer parecido físico con oraciones que son familiares..."

Este proceso se evidencia solo con algunas de las palabras o términos técnicos que lógicamente son en su gran mayoría los de más frecuente uso entre los hablantes que comparten ese argot. El hecho de formar o derivar palabras a partir de otras de un idioma extranjero es algo que no puede pasar desapercibido ante los lingüistas ya que es un medio para entender mejor los procesos lingüísticos en acción en nuestra época y 
Revista Hechos y Proyecciones del Lenguaje. No. 12. Universidad de Nariño -UNED. (ISSN: 0121-3350). (pp. 25-34). 2003.

evidenciar lo que diacrónicamente se configura en la lingüística histórica.

Los siguientes son algunos ejemplos con los cuales se puede evidenciar el proceso de cambio en el léxico y el repertorio lingüístico de los hablantes de un idioma afectado por la introducción de la tecnología. Para esto se tiene el proceso en dos aspectos: uno de formación lexical, y el otro de retroalimentación semántica.

\section{Formación lexical}

\subsection{Préstamo}

En este productivo proceso lingüístico moderno (Yule, 1984), hay una serie de términos, por ejemplo, que no tienen equivalentes en español (lógicamente, se sabe lo que son si los parafrasea) y que a pesar de esta aparente 'deficiencia', los usuarios de estos de ninguna manera se ven impedidos para tomar términos de la lengua extranjera. Los que siguen son algunos ejemplos de vocablos del inglés en informática cuyo equivalente en español en la mayoría de los casos no existe, recurriéndose al uso del mismo de la lengua extranjera.

hardware
software
prompt
modem
BASIC
bit
byte
cobol
debug
RAM
ROM
stack
ascii
pixel
abort

Es indudable que uno de los procesos más productivos en la nueva formación o introducción de vocabulario es el que se hace como préstamo de un idioma a otro. A través de la historia, el español se ha venido 'enriqueciendo' de extranjerismos (también como en los demás idiomas dada la influencia del inglés) que aceptados o no, son usados a diario por la gente. Un tipo especial de préstamo puede ser el de la traducción o calco. En este proceso ocurre una traducción directa de las palabras del 
Revista Hechos y Proyecciones del Lenguaje. No. 12. Universidad de Nariño -UNED. (ISSN: 0121-3350). (pp. 25-34). 2003.

idioma Inglés al español como el caso de algunos términos presentados anteriormente y que cuyo uso quizá no sea el más indicado. Ej.: enter (introducir), por 'entrar'; run (ejecutar) por 'correr'.

\subsection{Traducción por falsa analogía}

Otro grupo de términos son lo que se 'prestan' (dada su morfología) para que los usuarios incurran en una traducción equivocada debido a una falsa analogía morfológica que sugiere su significado en español.

$$
\begin{aligned}
& \text {-file (archivo) por 'fila' } \\
& \text {-save (grabar) por 'salvar' } \\
& \text {-type (teclear) por 'tipiar' } \\
& \text {-library (biblioteca) por librería }
\end{aligned}
$$

La famosa falsa analogía de library por 'librería' ya no es tan falsa, al menos en el área de la computación. En forma contundente se enfatizaba en las clases de inglés sobre el peligro de traducir de acuerdo a la insinuación de significado que daba la forma escrita. Es decir, los casos de library (biblioteca) por librería; exit (salida) por éxito; success (éxito) por suceso; lecture (conferencia) por lectura, etc. son utilizados con alguna frecuencia en documentos informales.

Sin duda alguna que esta advertencia se pasó por alto por parte de los traductores del inglés al español en informática y computación (a quienes, en parte, se imputaría el origen de este proceso de cambio) puesto que en este campo library ya se conoce y se acepta como 'librería': ej. Library of commands, 'librería de comandos', (como consta en la literatura técnica del campo) y no como se esperaría 'biblioteca de comandos'. De lo que podemos estar seguros es que esta traducción que marca una nueva denotación ha causado cambio en la significación de los dos términos.

\subsection{Acuñamiento}

Otra serie de palabras como que conllevan un proceso interesante desde el punto de vista lingüístico, como el descrito en Yule, (1984) se relaciona con lo que se denomina acuñamiento de nuevos términos de vocabulario a partir de otro de un idioma extranjero debido tal vez a la carencia de un equivalente definido, (ej. Check, chequear).

En informática este proceso refleja la actividad lingüística como un mecanismo de la producción en vista de la necesidad de acomodarse lexicalmente al vocabulario del nuevo contexto. 
Revista Hechos y Proyecciones del Lenguaje. No. 12. Universidad de Nariño -UNED. (ISSN: 0121-3350). (pp. 25-34). 2003.

Algunos ejemplos típicos son:

a. debug: como verbo que equivale a corregir un programa de los errores de programación, sea cual fuere el lenguaje que se utilice (bug significa 'insecto' y se relaciona como connotación con 'molestia', 'molestar') ha producido "debugear" en español. Lógicamente la pregunta aquí sería "¿y por qué no decir corregir?" La respuesta no es fácil. Tal vez por la necesidad de sentirse identificado con el medio específico en el que el usuario se desenvuelve, necesidad de destacarse en el medio, la moda tecnológica en el empleo de términos diferentes, prestigio, etc. Tampoco se descartaría la identificación con un grupo específico, área del conocimiento, sofisticación, distinción, etc.

b. type: equivalente de 'escribir a máquina' en computación (mecanografiar) 'escribir mediante el teclado', ha pasado al Español como "tipiar".

No se puede decir "escribir" por razones de ambigüedad, o "escribir a máquina" porque implica dos términos más, lo que lo hace preferible 'tipiar'.

Este puede ser el caso de palabras (sobre todo verbos) que en inglés solo requieren una sola unidad léxica o palabra para un significado que en español se requeriría de más de dos. Un ejemplo concreto fuera del campo de la computación lo podemos encontrar con circle, o sea, como verbo parafraseado 'encerrar en un círculo'. No ha habido ninguna forma evidente de uso para que la gente utilice "circular" o "encircular", a pesar de que sucede en traducciones dudosas.

c. return: Otro caso es el de return que gramaticalmente no es ni siquiera verbo en Inglés sino un simple e inofensivo sustantivo que equivale al nombre de una tecla del computador y a la acción que esta realiza aceptando la introducción de datos mediante el teclado o con la que se requiere la respuesta del computador (análogo al 'retorno' de carro en la máquina de escribir). Esto ha producido "returnear" (o 'dar' return) en Español, o sea, el acto de oprimir la tecla denominada return.

\section{Retroalimentación sintáctico-semántica}

Este quizás sea el caso más interesante en el que se puede constatar cómo la gente puede alterar las reglas de gramática, sintaxis y semántica, realizando un cambio no solo semántico sino también sintáctico que conlleva a la asimilación semántica a otras palabras en español.

Se tiene el caso de la traducción que se ha hecho de los términos enter y run como 
Revista Hechos y Proyecciones del Lenguaje. No. 12. Universidad de Nariño -UNED. (ISSN: 0121-3350). (pp. 25-34). 2003.

'entrar' y 'correr' respectivamente.

A simple vista el lector diría "pero si eso es precisamente lo que traduce enter y run en español". En realidad esa sería la traducción que se daría según nuestro conocimiento inicial de la implicación semántica y sintáctica de los términos. El problema sin embargo radica en el entorno lingüístico donde ocurren.

De ser así no habría problema en entender la traducción de:

-he enters the room

-he runs the $400 \mathrm{mts}$. hurdles

como,

-'el entra al cuarto'

-'el corre los 400 mts. vallas'

Sin embargo, este no sería el mismo caso de la traducción de enter y run en el argot de la informática:

-he enters the data

-he runs the program

para traducirlos como:

-'el entra los datos' (a)

-'el corre el programa' (b)

No obstante este es el uso habitual que da en informática. Sería apropiado (sin tener ninguna clase de prescripción en mente) el uso de los verbos entrar y correr en estos entornos sintácticos. Sin embargo, el problema es de dos clases. El primero la inclusión de una traducción para verbos intransitivos en entornos transitivos o sea en los que se haga evidente el uso del objeto directo, y el segundo, el problema semántico que acarrea el uso de verbos que denotan acciones físicas diferentes, o sea, 'entrar' por 'introducir' algo, y 'correr' por 'ejecutar' o poner en marcha algo. Los usuarios en el campo de la informática utilizan esta estructura sin problema de interferencia.

Por ejemplo. No habría problema en los casos: 
Revista Hechos y Proyecciones del Lenguaje. No. 12. Universidad de Nariño -UNED. (ISSN: 0121-3350). (pp. 25-34). 2003.

-Jaime introduce los datos

-Jaime ejecuta el programa

Introducir y ejecutar (verbos transitivos) aceptan los objetos directos 'datos' y 'programa', a diferencia de los verbos anteriormente mencionados 'entrar' y 'correr' (normalmente intransitivos en español) Podemos ver el efecto de forma y sentido que se produce si utilizáramos verbos intransitivos con complementos directos en oraciones tales como:

-Jaime sale los datos

-Jaime duerme la cama

Una sensación de perplejidad sería evidente (como muchos estudiantes nuevos lo hacen saber) en alguien nuevo o ajeno, en el peor de los casos, al campo de la tecnología de la información, informática y computación que no comparta este conocimiento y que escuche:

-corramos el programa

-entremos el comando

Pero no solo es el hecho de desconocer el vocabulario sino el hecho de que el oyente puede intentar hacer sentido de lo que oye lo cual sería algo completamente diferente a lo que en realidad se trata de comunicar.

Esto sería evidente si los oyentes no estuvieran informados del contexto físico (dónde y qué se disponen a hacer los hablantes) Sin embargo, podrían salir de dudas si alguien le dijera que 'correr' equivale a 'ejecutar' o 'poner en marcha un programa' en el computador para que este inicie un proceso. Y, que 'entrar' es 'introducir' mediante el teclado la información en el computador para que éste lo procese.

El punto de discusión aquí, con base a lo que inicialmente se ha podido analizar no es el de aceptar o rechazar el uso de traducciones como 'entrar' y 'correr como equivalentes de enter y run del inglés respectivamente. El interés aquí es puramente lingüístico. El hecho de importancia es que se pueda estar ante un proceso cambio y de alteración sintáctico-semántica debido a esta clase de usos por la exposición, interacción y despliegue continuo de los términos técnicos por parte de los usuarios en estos campos tecnológicos. 
Revista Hechos y Proyecciones del Lenguaje. No. 12. Universidad de Nariño -UNED. (ISSN: 0121-3350). (pp. 25-34). 2003.

¿Podría ocurrir que el verbo 'entrar' como tal se vea afectado diacrónicamente por el uso en un determinado argot o jerga para que se añada el rasgo de transitividad en determinados contextos? ¿Constituiría este un caso de creación, adaptación o evolución lingüísticas? Se podría decir que sí y se podría volver usual oír decir:

-Entre la moneda por el orificio.

-Corra la aplicación, de prisa!

Un gran caudal de alteración léxica, sintáctica y semántica se podría estar produciendo en esta época, en el grupo exclusivo de la gente entendida en la informática y la computación. Sin embargo, al paso que este campo está impactando en los diferentes dominios de la sociedad y del diario vivir no se entrevén impedimentos para que esta influencia se extienda dando paso a cambios más drásticos en situaciones de la vida diaria.

\section{Conclusión}

Se puede asegurar que muchos de los términos introducidos por la tecnología de la Información son el resultado de un proceso productivo mediado por el uso arbitrario que hacen los hablantes de este campo. Muchos de esos términos no han sido aprobados por la Real Academia de la Lengua Española (la gran mayoría), pero que continuarán circulando en la lengua con alguna o ninguna transformación de su forma original en inglés (forma y sentido). De todos modos se está en presencia de procesos lingüísticos históricos que marcarán el camino diacrónico del léxico especializado.

\section{Bibliografía}

Allan, K. (1986). Linguistic Meaning. New York: Routledge \& Kegan Paul Inc.

Chomsky, N. 1974: 'Topics in the Theory of Generative Grammar' in The Philosophy Of Language. J.R. Searle (ed.). Oxford Readings in Philosophy. Oxford University Press. Oxford.

Yule, G. (1984). The Study of Language. Cambridge: Cambridge University Press. 
Revista Hechos y Proyecciones del Lenguaje. No. 12. Universidad de Nariño -UNED. (ISSN: 0121-3350). (pp. 25-34). 2003.

\begin{abstract}
ANEXO
GLOSARIO DE LOS ALGUNOS TÉRMINOS COMUNES CON CAMBIO FORMA-SENTIDO DEL INGLÉS EN AL ESPAÑOL EN EL ARGOT DE LA INFORMÁTICA
\end{abstract}

\begin{tabular}{|c|c|c|c|}
\hline $\begin{array}{l}\text { TÉRMINO } \\
\text { (en inglés) }\end{array}$ & NATURALEZA & $\begin{array}{l}\text { EQUIVALENCIA } \\
\text { (en español) }\end{array}$ & USO COMUN \\
\hline ABORT & Verbo & Interrumpir & Abortar \\
\hline ACCESS & Verbo & Tener acceso a & accesar \\
\hline ALPHANUMERIC & Adjetivo & alfanumérico & alfanuméricc \\
\hline APPLICATION & Sustantivo & Producto & Aplicación \\
\hline $\begin{array}{l}\text { ASCII } \\
\text { (pronunciado ASKI) }\end{array}$ & Adjetivo & Código ASCII & ASCII \\
\hline BAUD & Sustantivo & Baudio & Baudio \\
\hline BIT & Sustantivo & Bitio o bit & Bit \\
\hline ВООТ & Verbo & Iniciar & Arrancar \\
\hline BUG & Sustantivo & Error & Bug \\
\hline BYTE & Sustantivo & Byte & Byte \\
\hline CHARACTER & Sustantivo & Símbolo & Caracter \\
\hline CHIP & Sustantivo & Chip & Chip \\
\hline COMMAND & Sustantivo & Instrucción & Comando \\
\hline COMPILER & Sustantivo & Compilador & Compilador \\
\hline CURSOR & Sustantivo & Cursor & Cursor \\
\hline DATA & Sustantivo & Datos & Dato \\
\hline DEFAULT & Adjetivo & Corriente & $\begin{array}{l}\text { Defecto, } \\
\text { defecto }\left(^{*}\right)\end{array}$ \\
\hline DISK & Sustantivo & Disco & Disco \\
\hline DRIVE & Sustantivo & Unidad de disco & Drive \\
\hline ENTER & Verbo & Introducir & $\frac{\text { Entrar }}{\text { (s.) }}$ \\
\hline FILE & Sustantivo & Archivo & fila \\
\hline FLOPPY DISK & Sustantivo & Disco Flexible & Disco floppy \\
\hline FORMAT & Verbo & Dar formato & Formatear \\
\hline HARD DISK & Sustantivo & Disco Duro & Disco duro \\
\hline HARDWARE & Sustantivo & Hardware & Hardware \\
\hline ICON & Sustantivo & Icono & Icono \\
\hline LIBRARY & Sustantivo & Biblioteca & Librería \\
\hline INPUT & Sustantivo & Entrada & Input \\
\hline INTERFACE & Sustantivo & Interfaz & Interface \\
\hline
\end{tabular}


Revista Hechos y Proyecciones del Lenguaje. No. 12. Universidad de Nariño -UNED. (ISSN: 0121-3350). (pp. 25-34). 2003.

$\begin{array}{llll}\text { JOYSTICK } & \text { Sustantivo } & \text { Control manual } & \text { Joystick } \\ \text { LOAD } & \text { Verbo } & \text { Cargar } & \text { Cargar } \\ \text { MENU } & \text { Sustantivo } & \text { Menú } & \text { Menú } \\ \text { MODEM } & \text { Sustantivo } & \text { Modem } & \text { Modem } \\ \text { MOUSE } & \text { Sustantivo } & \text { Ratón, Mouse } & \text { Mouse, ratón }\left(^{*}\right) \\ \text { OUTPUT } & \text { Sustantivo } & \text { Salida } & \text { Salida } \\ \text { PASSWORD } & \text { Sustantivo } & \text { Clave } & \text { Password, clave } \\ & & & \left({ }^{*}\right) \\ \text { PIXEL } & \text { Sustantivo } & \text { Pixilio } & \text { Pixel } \\ \text { PRINTER } & \text { Sustantivo } & \text { Impresora } & \text { Impresora } \\ \text { RAM } & \text { Sustantivo } & \text { Memoria RAM } & \text { Memoria Ram } \\ \text { ROM } & \text { Sustantivo } & \text { Memoria ROM } & \text { Memoria Rom } \\ \text { RUN } & \text { Verbo } & \text { Ejecutar } & \text { Correr } \\ \text { SAVE } & \text { Verbo } & \text { Grabar } & \text { Guardar, salvar } \\ \text { SOFTWARE } & \text { Sustantivo } & \text { Software } & \text { Software } \\ \text { TRACK } & \text { Sustantivo } & \text { Pista } & \text { Track, pista } \\ \text { TYPE } & \text { Verbo } & \text { Teclear } & \text { Tipiar }\end{array}$

$\left(^{*}\right)$ Se utiliza indistintamente y con bastante frecuencia.

Los términos subrayados son los que causarían conflicto en forma y sentido, especialmente para alguien fuera del medio informático.

Jorge E. Benavides, Profesor Asociado, adscrito al Departamento de Lingüística e Idiomas. Director del grupo de Interés C.A.L.L. Computer-Assisted Language Learning en ASOCOPI (www.udenar.edu.co/call); Director del grupo interdisciplinario de investigación TEKINED (www.udenar.edu.co/tekined); Tecnología de la Información en Educación. Miembro del grupo de investigación CURRICULO y UNIVERSIDAD (www.udenar.edu.co/curricuni). 